\title{
Unternehmenstheorie. Oh wie langweilig, braucht doch kein Mensch! Oder?
}

\author{
Thomas Hutzschenreuter*
}

Was ist ein Unternehmen? Die allermeisten meinen, die Antwort sei doch klar, vor allem aber unbedeutend. Ist doch ohnehin nur eine Definition. Wozu sich damit beschäftigen? Heute geht es doch um viel wichtigere Dinge. Digitalisierung, Nachhaltigkeit, Antiglobalisierung und so weiter. Heute geht es doch nicht um Theorie, sondern um Anwendungsorientierung. Die Betriebswirtschaftslehre sei doch eine anwendungsorientierte Disziplin, die braucht doch keine eigene Theorie, sondern sie holt sich ihre Theorien aus anderen Feldern und schafft auf dieser Basis Anwendungen für das Management. So oder so ähnlich bläst gegenwärtig der Wind Land auf und Land ab durch die Betriebswirtschaftslehre. Ich setze noch eins drauf. Vor kurzem, Festveranstaltung einer betriebswirtschaftlichen universitären Hochschule. Festrede. Schlussappell des Festredners an die Professor:innen der Fakultät. Trommelwirbel: „Liebe Betriebswirte, ich bitte Sie, bleiben Sie Handwerker, sie müssen nicht auch noch Wissenschaftler werden!" Pauke. Begeisterter Applaus der Mehrheit der Anwesenden. Prost!

\section{Was ist ein Unternehmen? Die Antwort ist eben nicht klar.}

Die Antwort auf diese Kernfrage der Theorie des Unternehmens ist eine Art Brille, durch die man sieht. Je nachdem, ob man eine Brille und welche Brille man aufhat, sieht man anders und man sieht etwas anderes. Die Brille richtet den Blick auf bestimmte Eigenschaften und wendet inn dabei von anderen Eigenschaften ab. Die Brille setzt etwas Bestimmtes ins Hellfeld und lässt anderes im Dunkeln. Für die Frage, was ich an und in einem Gegenstand sehe, wie ich ihn sehe und was ich nicht sehe, ist also entscheidend, welche Brille ich trage. Ronald H. Coase hat mit seinem 1937 erschienenen Artikel "The nature of the firm" die Diskussion zur Theorie des Unternehmens auf den Punkt gebracht. Er fragt in klarer Sprache und stringenter Argumentation danach, was ein Unternehmen ist und warum

Prof. Dr. Thomas Hutzschenreuter | Lehrstuhl für Strategisches und Internationales Management | Technische Universität München (TUM). 
Unternehmen existieren. Der damals vorherrschenden Diskussion lag die Idee zugrunde, dass Märkte als Quasi-Gegenstück zu Unternehmen primär existieren und die Existenz von Unternehmen derivativ von Märkten abzugrenzen sei. Coase schuf hierfür die Brille der Transaktionskosten und damit eine Sicht, die bis heute den Blick auf Unternehmen prägt. Coases Artikel hat der Diskussion um das Verständnis des Unternehmens ein eigenes Feld geschaffen. Die von Coase auf den Punkt gebrachte Diskussion um die Natur von Unternehmen ist bislang noch nicht zu einem Ende gekommen. Stattdessen stehen sich im Sinne von Thomas S. Kuhn mittlerweile größere Lager - man könnte sie als die Brillengestelle verstehen gegenüber. Es ist somit überhaupt nicht klar, was ein Unternehmen ist.

\section{Was ist ein Unternehmen? Die Antwort ist nicht unbedeutend.}

Die Antwort auf diese Frage bestimmt darüber, wie Unternehmer und Manager ihre Unternehmen führen. Und sie bestimmt über die Ausrichtung der Betriebswirtschaftslehre, denn mit dieser Antwort steht und fällt das Verständnis der Wissenschaft, deren Kerngegenstand Unternehmen sind. Mit anderen Worten, die Antwort auf die Frage, was ein Unternehmen ist, ist nicht unbedeutend, sondern das eigentliche Fundament der Betriebswirtschaftslehre. Man könnte so weit gehen, zu sagen, dass die Antwort auf die Frage, was ein Unternehmen ist, alles Weitere in der (wissenschaftlichen) Auseinandersetzung mit Unternehmen und damit alles Weitere in der Betriebswirtschaftslehre bestimmt. Man könnte auch sagen, dass dies die Anatomie der Betriebswirtschaftslehre ist.

\section{Erfolgreiche Anwendungsorientierung ohne Fundament ist unmöglich}

Aus diesem Grund weist eine Schlussfolgerung, die Betriebswirtschaftslehre brauche keine eigenen Theorien, weil sie eine anwendungsorientierte Disziplin sei, in die falsche Richtung. Wenn der Kerngegenstand der Betriebswirtschaftslehre nicht verstanden ist, wie sollen denn dann Aussagen zu seiner Gestaltung sinnvoll formuliert werden? Wenn der Kerngegenstand keiner eigenständigen Theorien bedarf, wieso braucht man dann ein eigenständiges Fach? Ohne ein auf den Kerngegenstand ausgerichtetes Fundament - keine erfolgreiche Anwendungsorientierung. Und ohne Kerngegenstand, der die Disziplin von allen anderen unterscheidet - keine Disziplin. Keine Unternehmenstheorie - keine Betriebswirtschaftslehre als Wissenschaft.

Unternehmer und Manager gestalten Zukunft. Ihr Handeln ist stets auf die Zukunft ausgerichtet. Das Verständnis, was ein Unternehmen ist, hängt davon ab, was über die Wissbarkeit der Zukunft angenommen wird. Und das Verständnis, was ein Unternehmen ist, bestimmt darüber, was die Betriebswirtschaftslehre leisten kann, vor allem aber auch, was sie nicht leisten kann. Dies bildet die Grundlage jeglicher Anwendungsorientierung der Betriebswirtschaftslehre. 


\section{Das Unternehmen als fortwährender Versuch}

Ist die Zukunft wissbar, lässt sich praktisches Unternehmenshandeln optimieren, die Möglichkeit von richtigen Entscheidungen existiert und Analytik und deduktive Logik dominieren. Optimalität im praktischen Unternehmenshandeln ist hingegen ausgeschlossen, wenn die Zukunft nicht wissbar ist. Zudem ist richtig oder falsch kein eindeutiges Kriterium. Neben Analytik und deduktive Logik treten individuelle, interpersonell nicht zwingend überprüfbare Imaginationen sowie Vertrauen und Überzeugungskraft jenseits „beweisbarer“ Gründe.

Alles Zukünftige ist unsicher. Es ist möglich, über Zukünftiges Prognosen, Vorhersagen, Vermutungen, Imaginationen, Planungen etc. anzustellen, aber eben nicht möglich, Wissen zu besitzen. Das zentrale unternehmenspraktische Problem ist, sichere Ausgaben tätigen zu müssen, um unsichere Einnahmen zu generieren; mit anderen Worten: es ist das Handeln unter individueller Unsicherheit. Wenn alles Zukünftige unsicher ist, wenn unternehmenspraktisches Handeln unter individueller Unsicherheit stattfindet, dann ist ein Unternehmen kein Gegenstand von Optimierung, sondern sein Wesen ist der Versuch. Dies ändert sich auch nicht mit fortschreitendem Bestehen eines Unternehmens. Das Unternehmen ist somit nicht nur ein Versuch zu Beginn seines Bestehens, sondern es ist ein fortwährender Versuch. Um den Versuch Unternehmen zu gestalten, bedient man sich institutioneller Gestaltungselemente, die das Unternehmen als Institution erscheinen lassen.

\section{Betriebswirtschaftslehre am Scheideweg?}

Die Betriebswirtschaftslehre als Einzelwirtschaftslehre hat im Kern das einzelne Unternehmen zum Gegenstand - seine Entstehung, sein Handeln und seine Entwicklung. Aus dem Verständnis des zentralen Objektes der Betriebswirtschaftslehre ergeben sich somit Implikationen für die Betriebswirtschaftslehre als Wissenschaft und damit für die Forschung innerhalb dieser Wissenschaft sowie die Bildung, die diese Wissenschaft vermitteln kann. Ein in seinen Kerncharakteristika anderes Verständnis vom Unternehmen zieht sogleich auch andere Implikationen für die Betriebswirtschaft als Ganzes sowie die betriebswirtschaftliche Forschung und Bildung nach sich.

Aus dem Verständnis des Unternehmens als Versuch und Institution folgen eine Reihe von Implikationen, die hier in Form von Thesen impulsartig und für die weitere Suche nach dem richtigen Weg appetitanregend genannt werden sollen. Ihre Diskussion wird darüber bestimmen, welchen Weg die Betriebswirtschaftslehre einschlägt:

(1) Die Betriebswirtschaftslehre als Wissenschaft kann weder wie die Physik noch wie die Medizin sein. Der Gegenstand der Betriebswirtschaftslehre ist fundamental anders, weswegen auch die Wissenschaft, die sich mit diesem völlig anderen Gegenstand auseinandersetzt, völlig anders sein muss.

(2) Die Betriebswirtschaftslehre kann nicht sagen, wie man Stroh zu Gold spinnt. In Anbetracht ihres mit Unsicherheit behafteten Gegenstands sollte die Betriebswirtschaftslehre bei ihren Adres- 
saten realistische Erwartungen an ihre Möglichkeiten aber auch ihre Grenzen ausprägen.

(3) Die Betriebswirtschaftslehre kann Beiträge zur Professionalisierung der Unternehmensführung, zur Regulierung sowie für den einzelnen Praktiker Hilfe zur Selbsthilfe leisten. Hierfür ist es zwingend notwendig, beim Problem des Praktikers zu starten.

Mögen die Professor:innen der Betriebswirtschaftslehre in erster Linie Wissenschaftler:innen sein, die ihren Kerngegenstand kennen und ernstnehmen. Das machte sie zu den besten Handwerkern, die man sich denken kann. Möge die Betriebswirtschaftslehre wieder in ihr Bergwerk einfahren und ihren Kern finden! Glück auf!

\section{Quelle}

Der Beitrag basiert auf „Das Unternehmen als Versuch und Institution. Ein Essay zum Kerngegenstand der Betriebswirtschaftslehre." von Thomas Hutzschenreuter erschienen im Verlag Franz Vahlen, München 2021. 